\title{
Energetics and monsoon bifurcations
}

\author{
Ashwin K. Seshadri ${ }^{1}$
}

Received: 22 January 2015 / Accepted: 19 March 2016 / Published online: 7 April 2016

(C) Springer-Verlag Berlin Heidelberg 2016

\begin{abstract}
Monsoons involve increases in dry static energy (DSE), with primary contributions from increased shortwave radiation and condensation of water vapor, compensated by DSE export via horizontal fluxes in monsoonal circulations. We introduce a simple box-model characterizing evolution of the DSE budget to study nonlinear dynamics of steady-state monsoons. Horizontal fluxes of DSE are stabilizing during monsoons, exporting DSE and hence weakening the monsoonal circulation. By contrast latent heat addition (LHA) due to condensation of water vapor destabilizes, by increasing the DSE budget. These two factors, horizontal DSE fluxes and LHA, are most strongly dependent on the contrast in tropospheric mean temperature between land and ocean. For the steady-state DSE in the box-model to be stable, the DSE flux should depend more strongly on the temperature contrast than LHA; stronger circulation then reduces DSE and thereby restores equilibrium. We present conditions for this to occur. The main focus of the paper is describing conditions for bifurcation behavior of simple models. Previous authors presented a minimal model of abrupt monsoon transitions and argued that such behavior can be related to a positive feedback called the 'moisture advection feedback'. However, by accounting for the effect of vertical lapse rate of temperature on the DSE flux, we show that bifurcations are not a generic property of such models despite these fluxes being nonlinear in the temperature contrast. We explain the origin of this behavior and describe conditions for a bifurcation to occur. This is illustrated for the case of the July-mean
\end{abstract}

Ashwin K. Seshadri

ashwin@fastmail.fm

1 Divecha Centre for Climate Change,

Indian Institute of Science, Bangalore 560012, India monsoon over India. The default model with mean parameter estimates does not contain a bifurcation, but the model admits bifurcation as parameters are varied.

Keywords Monsoon dynamics - Bifurcations $\cdot$ Nonlinear dynamics $\cdot$ Dry static energy fluxes $\cdot$ Energy balance models

\section{Introduction}

Large-scale monsoon circulations are driven by solar radiation (Kutzbach and Guetter 1984; Prell 1984; Clemens et al. 1991; Prell and Kutzbach 1992; Kutzbach and Liu 1997; Webster et al. 1998; Neff et al. 2001; Agnihotri et al. 2002), and sustained by internal feedbacks involving latent heat addition (LHA) during condensation of water vapor (Luo and Yanai 1983; Nitta 1983; Luo and Yanai 1984; Li and Yanai 1996; Webster et al. 1998). Dry static energy (DSE) budgets of monsoon regions carry evidence of these contributions during monsoon months. Specifically the contributions to DSE from radiation and LHA are compensated by the flux of DSE out of monsoon regions (Masson and Joussaume 1997; Trenberth et al. 2000). The importance of favorable energetics for monsoonal circulations makes possible one approach towards large-scale monsoon dynamics, that of studying the energy budget and its dynamical behaviors.

Studying the evolution of the DSE budget can be revealing if features of monsoon dynamics (e.g. stability, bifurcations) have analogues in aspects of the energy budget. Some contributions to this budget depend nonlinearly on DSE, and hence the study of its temporal evolution can shed light on nonlinear monsoon dynamics. This approach is not new. Previous authors have studied bifurcations 
(Levermann et al. 2009; Schewe et al. 2011) in one-dimensional ordinary differential equation (ODE) models of monsoons. Bifurcating literally means splitting into two and can occur in nonlinear differential equations, in which the uniqueness of steady-states as a function of parameters is not guaranteed (Iooss and Joseph 1997; Guckenheimer and Holmes 2002).

Simplified models of bifurcation behavior in monsoons have been developed, where the authors characterize the energy balance of monsoons implicitly by a single ODE (Levermann et al. 2009; Schewe et al. 2011), so that the steady-state condition is an algebraic equation. The steady state is a result of writing the expression for energy balance of the monsoon region, and using conservation laws and parameterizations to represent all other quantities, including winds and precipitation, in terms of the state variable (Levermann et al. 2009; Schewe et al. 2011). These authors have then examined solutions of the algebraic equation describing the monsoon steady state (Levermann et al. 2009; Schewe et al. 2011) to characterize nonlinear dynamics, and point out that a bifurcation to the solutions exists and describe its properties.

In the approach based on the DSE, as in Levermann et al. (2009) and in this paper, the explicit role of moisture is to change the DSE of the monsoon system. Moisture also plays an implicit role by influencing the vertical lapse rate of temperature, which, as will be shown, plays an important role in nonlinear dynamics of monsoon models based on the DSE balance. This effect has been neglected by Levermann et al. (2009). A completely different approach might be to consider the moist-static energy dynamics of the monsoon system, where the moist static energy is defined as DSE + latent heat content (Neelin and Held 1987). The moist static energy equation combines the DSE and moisture equations, and thinking in terms of this alternate variable would yield a different equation for describing the idealized monsoon, with possible differences in dynamical behavior. However, neither the DSE dynamics nor that of the moist static energy counterpart would be complete by itself. A more comprehensive approach would be to include dynamics of both moisture and DSE in the system of equations.

In an important study, Boos and Storelvmo (2016) consider explicitly the steady-state temperature and moisture equations in monsoon regions, combining them to yield a single polynomial equation for the system's steady-state. They also performed global climate model simulations for a wide range of forcings, finding no evidence of monsoon bifurcation. Like the present paper they note (Boos and Storelvmo 2016) that these previous simplified models of bifurcation (Levermann et al. 2009; Schewe et al. 2011) leave out a necessary term in monsoon dynamics corresponding to the static stability of the troposphere. Boos and
Storelvmo (2016) find that when accounting for this term's influence the seasonal-mean response of the monsoon to forcing becomes almost linear.

These and other previous studies have examined tipping points in monsoon systems (Zickfeld et al. 2005; Lenton et al. 2008; Levermann et al. 2009; Schellnhuber 2009; Schewe et al. 2011). The existence of a tipping point in energy balance models of the monsoon was first pointed out by Zickfeld et al. (2005). The model of Zickfeld et al. (2005) comprised four ODEs, describing evolution of temperature, specific humidity, and soil moisture in two layers. Although a saddle-node bifurcation was apparent from numerical analysis of the model, the origin of the bifurcation was not specified (Zickfeld et al. 2005) due to the complexity of that model. Mathematically, existence of tipping points is equivalent to a saddle-node bifurcation where stable and unstable steady states collide and annihilate each other (Iooss and Joseph 1997). Once a stable steady-state disappears at a bifurcation point, there is rapid movement towards a different steady state that is stable. Physically this would be experienced as rapid change, posing challenges to adaptation (Davis and Shaw 2001). Therefore the work of Zickfeld et al. (2005), by identifying a potential tipping point, characterized an important feature relevant to monsoon models. This line of work involving idealized modeling in the presence of nonlinearities is what led Levermann et al. (2009) to eventually identify nonlinear advection as the origin of bifurcation behavior in a highly simplified monsoon model.

The models of (Levermann et al. 2009; Schewe et al. 2011) contain a single ODE. Such a simplification is not always justified, and characterizing monsoon dynamics in this way by a single ODE representing the evolution of DSE assumes that other variables involved in characterizing DSE can be related algebraically to the state variable, and thus parameterized. This is valid only if the other parameterized variables adjust on timescales much shorter than characteristic times for the evolution of DSE. This condition is generally not satisfied, and including dynamical behaviors of these other variables would yield higherdimensional systems of ODEs (e.g. Zickfeld et al. 2005; Knopf et al. 2006). These larger systems need not have the same bifurcation characteristics as their one-dimensional counterparts, which typically involve averaging effects of some variables by treating them as known functions of the dynamical variable.

One factor that weighs in favor of simplifying the model to a one-dimensional system, for studying monsoon bifurcations, is that in a saddle-node bifurcation the eigenvalue of the linearized system approaches zero. This corresponds to the slowing down of the system (Scheffer et al. 2009). Hence near this regime it is possible that other variables adjust rapidly enough to be modeled as if they 
would respond instantly. In our case of a simplified monsoon model, following the one-dimensional examples of Levermann et al. (2009) and Schewe et al. (2011), winds and precipitation should adjust rapidly compared to evolution of the DSE variable. However making additional variables prognostic in the model generally alters the structure of coupling between variables, and therefore even the aforementioned slowing down cannot ensure that nonlinear dynamical behavior is preserved. Whether or not the bifurcation behavior of the simplest model mimics the behavior of more complex models, in which these other variables are allowed to be prognostic, can only be determined by studying these different models and comparing their dynamical behaviors.

Despite these limitations this paper considers bifurcation behavior of single-ODE monsoon models, describing evolution of DSE over monsoon regions with monsoonal-type circulations, involving convergence below and divergence aloft. Favorable energetics is essential for monsoonal circulations (Trenberth and Stepaniak 2003), and a single-ODE model provides a minimal conceptual and mathematical model for monsoon bifurcations. The reason for elaborating previous work despite the simplicity of their models (Levermann et al. 2009; Schewe et al. 2011) is that actual dependence of DSE fluxes on land-ocean temperature differences is qualitatively different, when accounting for effects of vertical variation in temperature on horizontal fluxes of DSE. The effect of considering the consequences of the resulting vertical gradients in DSE is different nonlinear dynamical behavior even in such idealized models so that, unlike Levermann et al. (2009), who did not consider the influence of vertical temperature variation, a bifurcation is not intrinsic to such models despite the nonlinearity of DSE flux. What is in fact required for a tipping point to occur is that the sensitivity of latent heat addition must be sufficiently strong to offset the effect of DSE flux and produce a bifurcation. When vertical gradients in DSE are included in the model, as they must be, this condition does not necessarily occur. Furthermore, in contrast to the findings of Boos and Storelvmo (2016) who argue that accounting for dry thermal stratification eliminates the bifurcation, we show that a bifurcation is still possible but is no longer generic to the model, and depends on further details. Therefore it is fitting to clarify the origin of bifurcation phenomena in one-dimensional models of idealized monsoons, as this paper seeks to do.

\section{Models}

\subsection{Box-model of dry static energy balance}

The box-model developed in this paper describes evolution of the DSE balance, in the presence of monsoon-type circulations that involve low-level convergence and upper-level divergence. The reverse case, with low-level divergence and upper-level convergence, is also considered. The control volume comprises the troposphere over a land region where the monsoon is present (Fig. 1). In the model, vertical temperature variation is treated as being linear in the height
Fig. 1 Control volume of tropospheric column over land in the model of dry static energy (DSE) balance. Surface pressure is $p_{0}$, overturning level is at pressure $p_{D}$, and the top of the control volume is approximated to be at zero pressure. Also shown are incoming and outgoing DSE fluxes $S_{\text {in }}^{f}$ and $S_{\text {out }}^{f}$ respectively, with $s_{L}$ and $s_{O}$ being the DSE densities within the control volume and at ocean boundaries respectively: a monsoonal circulation, with $x \equiv \bar{T}_{L}-\bar{T}_{O}-x_{0 w}>0$; b reversed circulation, with $x \equiv \bar{T}_{L}-\bar{T}_{O}-x_{0 w}<0$. Parameter $x_{0 w}$ is the temperature difference that corresponds to zero wind-speed (a)

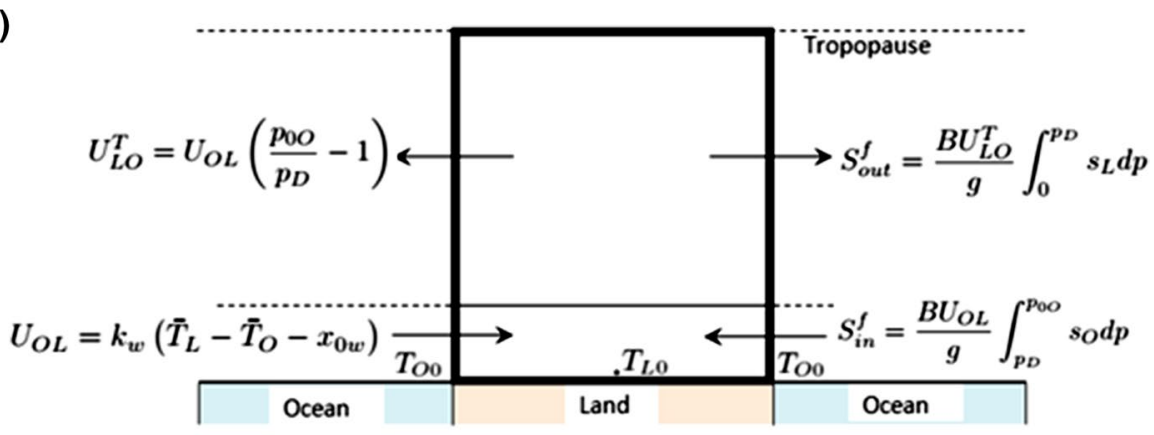

(b)

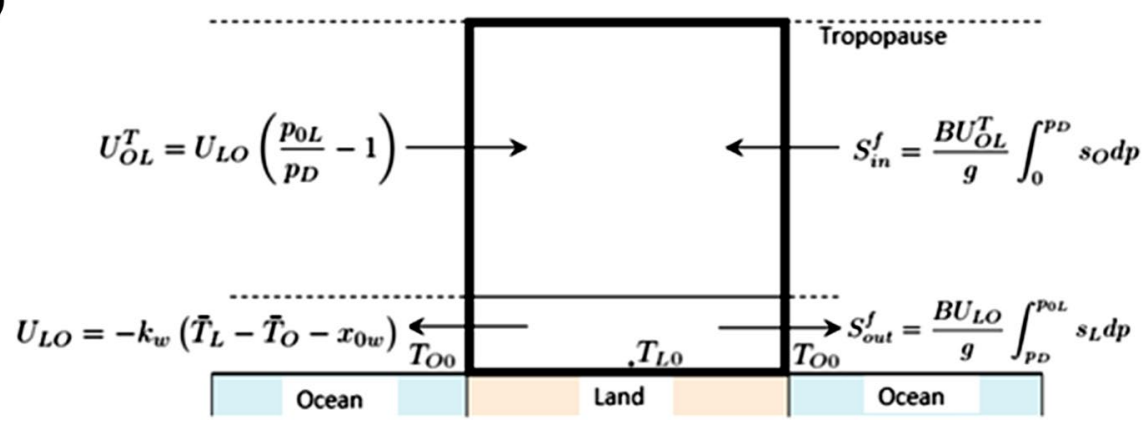


above the surface, so that $T(z)=T_{L 0}-\Gamma_{L} z$. Vertical variation in pressure is characterized by vertically uniform pressure scale-height $H_{S L}=R \bar{T}_{L} / g$, where $\bar{T}_{L}$ is mass-weighted mean temperature of the tropospheric column over land. As a result vertical pressure variation is described by $\ln \frac{p}{p_{0}}=-\frac{z}{H_{s L}}$, obtained by integrating the hydrostatic balance equation and applying the ideal gas law.

Following the treatment of Zickfeld et al. (2005) the control volume is surrounded by ocean, with fixed temperatures along the ocean boundary. A constant level called the overturning level $(\mathrm{OL})$ defined by pressure $p_{D}$ separates lower-level convergence into the control volume from upper-level divergence, during monsoon months. In case of reversed circulation, a different value of $p_{D}$ is taken to separate lower-level divergence from upper-level convergence into the control volume.

\subsubsection{Winds}

Circulation in the model is defined by the wind-speed below the overturning level, which is assumed independent of height below this level in the model, and parameterized by formula

$U_{O L}=k_{w}\left(\bar{T}_{L}-\bar{T}_{O}-x_{0 w}\right)=k_{w} x$

where $\bar{T}_{L}$ and $\bar{T}_{O}$ are mass-weighted mean temperatures of the tropospheric column over land and ocean respectively and $x_{0 w}$ is the temperature difference that corresponds to zero wind-speed. The variable $x$ is the state-variable of the model and related to the land-ocean temperature contrast. This model is broadly consistent with models of thermally direct flow from ocean to the atmosphere above a tropical continent (following Levermann et al. 2009; Boos and Storelvmo 2016). When $x=0$ the wind-speed $U_{O L}=0$ and the sign of $U_{O L}$ follows the sign of $x$.

The parameters in Eq. (1) are estimated in Sect. 3.2. It turns out that $x_{0 w}$ is negative, indicating that zero windspeed occurs at negative land-ocean temperature contrast. This is simply because much of the land-surface is above sea level, and the corresponding pressure-depths over which mass-weighted temperatures are estimated are different for land and ocean.

\subsubsection{DSE balance}

The evolution of DSE for an infinitesimal volume is described by

$\frac{\partial \rho s}{\partial t}=\rho g \frac{\partial\left(F^{R}+F^{S}\right)}{\partial p}+\rho Q_{L H}-\nabla_{H} \circ \rho s \vec{V}-\frac{\partial \rho s \omega}{\partial p}$

where $s=c_{p} T+g z$ is DSE per unit mass, $F^{R}$ is upward radiative flux, $F^{S}$ is upward sensible heat flux, $Q_{L H}$ is latent heating per mass, $\nabla_{H}$ is horizontal divergence, $\vec{V}$ is the horizontal velocity vector, and $\omega$ is vertical velocity in pressure coordinates.

\subsubsection{Left side of DSE balance}

The rate of change of DSE integrated over the control volume is $\frac{\partial}{\partial t} \int \rho s d V$. Using the relation $\rho d V=\rho d A d z=-(1 / g) d A d p$ and the definition for DSE $s=c_{p} T+g z$, the integral becomes

$$
\begin{aligned}
& \int \rho s d V=\frac{1}{g} \int d A \int_{0}^{p_{0 L}} s d p \\
& \quad=\frac{A}{g} \int_{0}^{p_{0 L}}\left(c_{p}\left(T_{L 0}-\Gamma_{L} H_{S L} \ln \frac{p_{0 L}}{p}\right)+g H_{S L} \ln \frac{p_{0 L}}{p}\right) d p
\end{aligned}
$$

where $A$ is the horizontal area of the control volume. To derive the above we have used relation $T(z)=T_{L 0}-\Gamma_{L} z$, and that $z=H_{S L} \ln \frac{p_{0 L}}{p}$. Equation (3) simplifies to $\int \rho s d V=\frac{A c_{p} p_{0 L}}{g}\left(T_{L 0}+\left(\Gamma_{D}-\Gamma_{L}\right) H_{s L}\right)$ where $\Gamma_{D}=g / c_{p}$ is the dry adiabatic lapse rate, and using $\lim _{p \rightarrow 0} p \ln p=0$. Using the relation $\bar{T}_{L}=T_{L 0}-\Gamma_{L} H_{S L}$ between columnmean temperature and pressure scale height (see "Appendix" for derivation), we can eliminate the surface temperature above to write $\int \rho s d V=\frac{A c_{p} p_{0 L}}{g}\left(\bar{T}_{L}+H_{s L} \Gamma_{D}\right)$, and differentiation yields

$\frac{\partial}{\partial t} \int \rho s d V=\frac{A c_{p} p_{0 L}}{g}\left(1+\frac{R}{c_{p}}\right) \frac{\partial \bar{T}_{L}}{\partial t}$

after substituting expressions for $\Gamma_{D}$ and $H_{S L}$. We have assumed that surface pressure is constant, at $p_{0 L}$. Dry static energy is directly proportional to the column mean temperature.

\subsubsection{Model of DSE flux}

With monsoonal circulation, involving lower-level convergence into the control volume and upper-level divergence, the incoming flux of DSE below the overturning level is

$S_{i n}^{f}=\int_{0}^{z_{D}} \rho s_{O} u_{O L} d z \int d B$

where $z_{D}$ is the height of the overturning level, $s_{O}$ is the DSE at the ocean boundary, $u_{O L}$ is the component of velocity directed into the atmospheric box and normal to its surface, and $d B$ is a length element along the boundary. Writing the integral of the length element $\int d B$ as $B$, assuming that below the overturning level $u_{O L}$ is constant and equal to $U_{O L}$, reparameterizing in terms of pressure for $S_{i n}^{f}=\frac{B U_{O L}}{g} \int_{p_{D}}^{p_{0 O}} s_{O} d p$, and substituting the parameterization for $U_{O L}$ and simplifying the integral yields 


$$
\begin{aligned}
S_{i n}^{f}= & \frac{B k_{w} c_{p}\left(p_{0 O}-p_{D}\right)}{g}\left(\bar{T}_{L}-\bar{T}_{O}-x_{0 w}\right) \bar{T}_{O} \\
& \times\left(\left(1+\frac{R}{c_{p}}\right)+\frac{R}{g}\left(\Gamma_{D}-\Gamma_{O}\right) \frac{p_{D}}{p_{0 O}-p_{D}} \ln \frac{p_{D}}{p_{0 O}}\right)
\end{aligned}
$$

where $p_{0 O}$ is surface pressure at ocean boundaries. We have omitted some steps in the derivation, for which the reader is referred to the Supplementary Information. Similarly the expression for outgoing flux of DSE from the box is

$S_{\text {out }}^{f}=\int_{z_{D}}^{z_{T}} \rho s_{L} u_{L O} d z \int d B=\frac{B U_{L O}^{T}}{g} \int_{0}^{p_{D}} s_{L} d p$

where $U_{L O}^{T}$ is the constant velocity of the divergent flow above the overturning level OL. The velocity $U_{L O}^{T}$ is related to $U_{O L}$ via the equation for continuity. The differential form of this equation is integrated over the volume of the box

$\int \frac{\partial \rho}{\partial t} d V+\int \nabla \circ(\rho u) \cdot d V=0$

and transforming to pressure coordinates and using the divergence theorem yields

$\frac{A}{g} \frac{\partial p_{0 L}}{\partial t}=\frac{B}{g}\left[U_{O L}\left(p_{0 O}-p_{D}\right)-U_{L O}^{T}\left(p_{D}-0\right)\right]$

We assume mass conservation within the control volume so that $p_{O L}$ is constant. Then $U_{L O}^{T}=U_{O L}\left(p_{0 O} / p_{D}-1\right)$. Substituting this into Eq. (7) and simplifying the integrals yields for outgoing flux of DSE

$$
\begin{aligned}
S_{\text {out }}^{f}= & \frac{B k_{w} c_{p}\left(p_{0 O}-p_{D}\right)}{g}\left(\bar{T}_{L}-\bar{T}_{O}-x_{0 w}\right) \bar{T}_{L} \\
& \times\left(\left(1+\frac{R}{c_{p}}\right)-\frac{R}{g}\left(\Gamma_{D}-\Gamma_{L}\right) \ln \frac{p_{D}}{p_{0 L}}\right)
\end{aligned}
$$

The difference between incoming and outgoing fluxes is

$$
\begin{aligned}
S_{\text {net }}^{f}(x) \equiv & S_{\text {in }}^{f}-S_{\text {out }}^{f} \cong-\frac{B k_{w} c_{p}\left(p_{0 O}-p_{D}\right)}{g} \\
& \times\left(\bar{T}_{L}-\bar{T}_{O}-x_{0 w}\right)\left(\left(\bar{T}_{L}-\bar{T}_{O}\right)\left(1+\frac{R}{c_{p}}\right)\right. \\
& \left.+\frac{R}{c_{p}}\left(f_{D O} k_{O} \bar{T}_{O} \ln \frac{p_{0 O}}{p_{D}}+k_{L} \bar{T}_{L} \ln \frac{p_{0 L}}{p_{D}}\right)\right)
\end{aligned}
$$

where $k_{O}=\left(\Gamma_{D}-\Gamma_{O}\right) / \Gamma_{D}, \quad k_{L}=\left(\Gamma_{D}-\Gamma_{L}\right) / \Gamma_{D}$, and $f_{D O}=p_{D} /\left(p_{0 O}-p_{D}\right)$ are positive quantities. The above expression is valid where $x=\bar{T}_{L}-\bar{T}_{O}-x_{0 w}>0$ with convergence below and divergence aloft.

In the opposite condition, the control volume takes in DSE from above the overturning level and vents from below. Then the DSE flux into the control volume above the overturning level is

$S_{\text {in }}^{f}=\int_{z_{D}}^{z_{T}} \rho s_{O} u_{O L} d z \int d B$

simplifying to

$$
\begin{aligned}
S_{i n}^{f}= & \frac{B k_{w} c_{p}\left(p_{0 L}-p_{D}\right)}{g}\left(\bar{T}_{O}-\bar{T}_{L}+x_{0 w}\right) \bar{T}_{O} \\
& \times\left(\left(1+\frac{R}{c_{p}}\right)-\frac{R}{g}\left(\Gamma_{D}-\Gamma_{O}\right) \ln \frac{p_{D}}{p_{0 O}}\right)
\end{aligned}
$$

using the relation $U_{O L}^{T}=U_{L O}\left(p_{0 L} / p_{D}-1\right)$ from mass-conservation (see Fig. 1). We recall that $p_{D}$ takes a different value in this condition and corresponding $p_{0 L}-p_{D}>0$. The DSE flux out of the control volume below the overturning level is

$S_{\text {out }}^{f}=\int_{0}^{z_{D}} \rho s_{L} u_{L O} d z \int d B$

simplifying to

$$
\begin{aligned}
S_{\text {out }}^{f}= & \frac{B k_{w} c_{p}\left(p_{0 L}-p_{D}\right)}{g}\left(\bar{T}_{O}-\bar{T}_{L}+x_{0 w}\right) \bar{T}_{L} \\
& \times\left(\left(1+\frac{R}{c_{p}}\right)+\frac{R}{g}\left(\Gamma_{D}-\Gamma_{L}\right) \frac{p_{D}}{p_{0 L}-p_{D}} \ln \frac{p_{D}}{p_{0 L}}\right)
\end{aligned}
$$

In this case, the difference between incoming and outgoing fluxes in Eqs. (13) and (15) is

$$
\begin{aligned}
S_{n e t}^{f}(x) \cong & \frac{B k_{w} c_{p}\left(p_{0 L}-p_{D}\right)}{g}\left(\bar{T}_{O}-\bar{T}_{L}+x_{0 w}\right)\left(\left(\bar{T}_{O}-\bar{T}_{L}\right)\right. \\
& \left.\times\left(1+\frac{R}{c_{p}}\right)+\frac{R}{c_{p}}\left(k_{O} \bar{T}_{O} \ln \frac{p_{0 O}}{p_{D}}+f_{D L} k_{L} \bar{T}_{L} \ln \frac{p_{0 L}}{p_{D}}\right)\right)
\end{aligned}
$$

where $f_{D L}=p_{D} /\left(p_{0 L}-p_{D}\right)>0$. The explicit manner in which the DSE flux in Eqs. (11) and (16) for the two respective cases depends on the state variable $x=\bar{T}_{O}-\bar{T}_{L}-x_{0 w}$ is shown in Sect. 2.2, where the dynamics of this flux is described further.

\subsubsection{Governing equation of the model}

Integrating Eq. (2) over the control volume and substituting Eqs. (4), and (11) or (16) depending on whether surface winds are landward or oceanward, yields our governing equation

$$
\begin{aligned}
G_{T}(x) \frac{d x}{d t}= & F(x) \equiv\left(F_{n e t}^{S W}(x)+F_{N e t}^{L W}(x)+F_{B}^{S}(x)\right) \\
& +F_{L H}(x)+\frac{1}{A} S_{\text {net }}^{f}(x)
\end{aligned}
$$


where $x \equiv \bar{T}_{L}-\bar{T}_{O}-x_{0 w}$ is the state variable characterizing the system and $G_{T}(x)=G_{T}=\frac{c_{p} p_{0 L}}{g}\left(1+\frac{R}{c_{p}}\right) . F_{n e t}^{S W}$ and $F_{N e t}^{L W}$ are respectively the shortwave and longwave differences between upward radiative fluxes at the bottom and top of the $\mathrm{CV}, F_{B}^{S}$ is sensible heat flux at the bottom, $F_{L H}=\left(p_{0 L} / g\right) Q_{L H}$ is the effect of LHA in units of flux, and $S_{\text {net }}^{f}$ is net horizontal DSE flux. Positive values of $x$ correspond to monsoonal circulations with landward surface winds. The value of $x$ can vary in the model only due to changes in column-mean temperature over land, i.e. $\bar{T}_{L}$, which is the only varying factor in the DSE of the control volume. Please compare Eq. (4) and the left side of the governing Eq. (17). The other parameters involved in the definition of $x$ are assumed to be fixed. This corresponds to the assumption of constant ocean boundary conditions and fixed value of $x_{0 w}$, which are estimated in Sect. 3 .

\subsubsection{Parameterization of LHA}

Effects of moisture condensation via LHA are represented in the term $F_{L H}$, parameterized by

$F_{L H}=k_{L H}\left(\frac{\bar{T}_{L}-\bar{T}_{O}-x_{m}}{\bar{T}_{O}}\right)^{\alpha_{L H}}=k_{L H}\left(\frac{x+x_{0 w}-x_{m}}{\bar{T}_{O}}\right)^{\alpha_{L H}}$

with $x_{m}$ being the lowest value of $\bar{T}_{L}-\bar{T}_{O}$. Then at the minimum value of $\bar{T}_{L}-\bar{T}_{O}$ the value of $x+x_{0 w}-x_{m}$ is $\bar{T}_{L}-\bar{T}_{O}-x_{0 w}+x_{0 w}-x_{m}=0$ so that the latent heat addition is zero. The relation also depends on constant parameter $\alpha_{L H}$. Parameters $k_{L H}$ and $\alpha_{L H}$ are estimated in Sect. 3.2.

\subsection{Dynamics of DSE flux}

Let us examine the dynamics of the DSE flux in our model under monsoonal and reversed circulations. Under monsoonal circulation, the DSE flux is calculated as

$S_{\text {net }}^{f} \equiv S_{\text {in }}^{f}-S_{\text {out }}^{f}=-k_{a}\left(p_{0 O}-p_{D}\right) x\left(c_{01}+c_{11} x\right)$

where $k_{a}=B k_{w} c_{p} / g, c_{01}=\left(1+\frac{R}{c_{p}}\right) x_{0 w}+\frac{R}{c_{p}}\left(f_{D O} k_{O} \bar{T}_{O} \ln \right.$ $\left.\frac{p_{0 O}}{p_{D}}+k_{L}\left(\bar{T}_{O}+x_{0 w}\right) \ln \frac{p_{0 L}}{p_{D}}\right)>0 \quad$ and $\quad c_{11}=1+\frac{R}{c_{p}}(1+$ $\left.k_{L} \ln \frac{p_{0 L}}{p_{D}}\right)>0$.

In case of reversed circulation the flux is

$S_{\text {net }}^{f} \equiv S_{\text {in }}^{f}-S_{\text {out }}^{f}=k_{a}\left(p_{0 L}-p_{D}\right) x\left(c_{02}+c_{12} x\right)$

where $c_{02}=\left(1+\frac{R}{c_{p}}\right) x_{0 w}-\frac{R}{c_{p}}\left(k_{O} \bar{T}_{O} \ln \frac{p_{0 O}}{p_{D}}+f_{D L} k_{L}\left(\bar{T}_{O}+\right.\right.$ $\left.\left.x_{0 w}\right) \ln \frac{p_{0 L}}{p_{D}}\right)<0$ and $c_{12}=1+\frac{R}{c_{p}}\left(1-f_{D L} k_{L} \ln \frac{p_{0 L}}{p_{D}}\right)>0$.

We note that $p_{D}$ in general takes different values in the two cases.
From Eq. (19), which applies if $x>0$ when surface winds are landward, the DSE flux is negative in case of monsoonal circulation. Therefore DSE flux in this case reduces the DSE of the control volume. From Eq. (20), applying when $x<0$, or surface winds are oceanward, the DSE flux is positive under reversed circulation. The flux then increases the DSE. Thus nonzero DSE flux tries to eliminate surface winds in the model but in general cannot, due to the other contributions to the DSE balance in Eq. (17). In this sense the DSE flux has a stabilizing influence on the DSE balance and monsoonal circulations.

Bifurcation behavior is influenced by the sensitivity of the DSE flux at the zero-point of $x$, when winds reverse, for reasons that will become clear in Sect. 2.3. In this respect we note that, at $x \rightarrow 0^{+}$, for $x$ approaching 0 from above, the sensitivity is $\partial S_{\text {net }}^{f} / \partial x \cong-k_{a}\left(p_{0 O}-p_{D}\right) c_{01}$, which is strictly negative, i.e. nonzero. The DSE flux has a nonzero linear contribution, and hence nonzero slope at $x=0$. This influences bifurcation behavior of the model, as will be shown.

Here we merely discuss the source of this behavior, wherein the DSE flux has nonzero slope at the origin. The term $\partial S_{\text {net }}^{f} / \partial x<0$ as long as $p_{0 O}>p_{D}$ and $c_{01}>0$. This requires that $p_{0 O}>p_{D}$, i.e. overturning occurs at some height in the troposphere. It also requires that

$$
\begin{aligned}
c_{01}= & \left(1+\frac{R}{c_{p}}\right) x_{0 w}+\frac{R}{c_{p}}\left(f_{D O} k_{O} \bar{T}_{O} \ln \frac{p_{0 O}}{p_{D}}\right. \\
& \left.+k_{L}\left(\bar{T}_{O}+x_{0 w}\right) \ln \frac{p_{0 L}}{p_{D}}\right)>0
\end{aligned}
$$

The last condition in turn requires that $k_{O}$ be strictly positive, because the dominant contribution is from this term, as shown in Sect. 4.1. For $k_{O}$ to be strictly positive requires that the lapse rate over ocean must be smaller than dry-adiabatic. These conditions are satisfied and hence the rate of decrease with land-ocean temperature contrast of the DSE flux is strictly negative.

The above relation is easily explained. Consider the equation for DSE per unit mass $s=c_{p} T+g z$, so that its rate of change with height is $\partial s / \partial z=c_{p}\left(\Gamma_{D}-\Gamma\right)>0$. DSE per unit mass increases with height as long as temperature decreases at a rate smaller than the dry adiabatic lapse rate. Mass-conserving monsoonal circulations, with convergence below and divergence above, therefore export DSE. Even when the circulation strength is close to zero, corresponding to limit $x \rightarrow 0^{+}$, the difference between mean DSE exported by the upper divergent circulation and that imported by the lower converging circulation depends linearly on circulation velocity. Hence the sensitivity of DSE flux is strictly negative, so that $\partial S_{\text {net }}^{f} / \partial x<0$. This simple physical argument shows that nonzero $\partial S_{\text {net }}^{f} / \partial x$ is a necessary feature of such circulations, and not merely an artifact of the simplifications in the above model. 


\subsection{Condition for bifurcation}

The condition for bifurcation follows from the implicit function theorem (Spivak 1965; Crawford 1991; Iooss and Joseph 1997; Guckenheimer and Holmes 2002). This general result specifies the condition for a steady state of a model to uniquely persist in the local neighborhood of a solution. Consider solution $\vec{y}(t ; \vec{\mu})$ to an autonomous system of ODEs given by equations $d \vec{y} / d t=g_{\vec{\mu}}(\vec{y})$ that depends on parameter set $\vec{\mu}$. For $\vec{\mu}=\vec{\mu}_{0}$, a steady state $\vec{y}_{0}$ satisfies $d \vec{y}\left(\vec{y}_{0}\right) / d t=0$. With $\vec{\mu}$ varied smoothly about $\vec{\mu}_{0}$, the steady-states in the neighborhood of $\vec{y}_{0}$ are unique if the Jacobian derivative of $g_{\vec{\mu}}(\vec{y})$ with respect to $\vec{y}$ has nonzero determinant when evaluated at $\vec{\mu}=\vec{\mu}_{0}$ and $\vec{y}=\vec{y}_{0}$. In our one-dimensional monsoon model of Eq. (17) the equivalent condition for a unique solution is $\partial \dot{x} / \partial x \neq 0$, where $\dot{x} \equiv d x / d t$.

Therefore the condition for a bifurcation is the opposite, i.e. $\partial \dot{x} / \partial x=0$. In graphical terms, if we were to plot $\dot{x}$ versus $x$, bifurcation points are where the slope of this graph is zero. Differentiating Eq. (17) with respect to $x$, we obtain $\left(\partial G_{T}(x) / \partial x\right) \dot{x}+G_{T}(x)(\partial \dot{x} / \partial x)=\partial F(x) / \partial x$. Using the fact that $\dot{x}=0$ at a steady-state solution this yields $G_{T}(x)(\partial \dot{x} / \partial x)=\partial F(x) / \partial x$, and hence the condition for bifurcation of steady state solutions is $\partial F(x) / \partial x=0$. With our model it so happens that $\partial G_{T}(x) / \partial x=0$, but the above discussion shows why the bifurcation condition would be $\partial F(x) / \partial x=0$ even in models where this is not the case. Where equality $\partial F(x) / \partial x=0$ is met, a bifurcation occurs and the solution splits (i.e bifurcates).

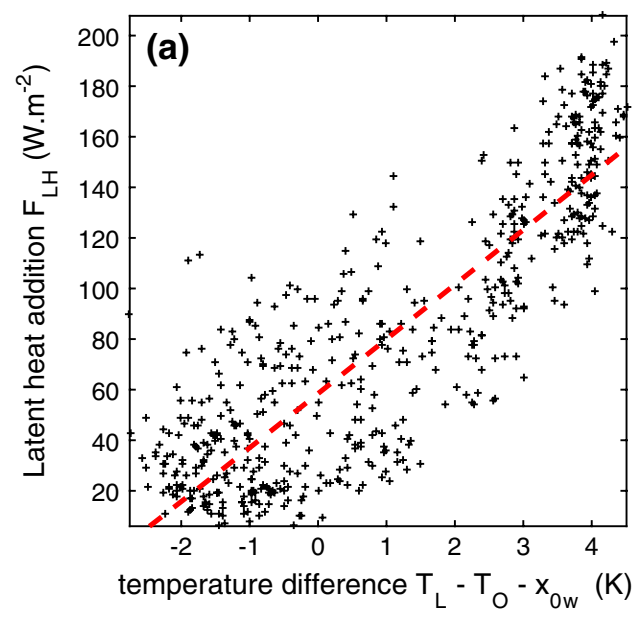

Fig. 2 Parameterization of latent heat addition (LHA): a graph of LHA $F_{L H}$ versus the temperature difference $\bar{T}_{L}-\bar{T}_{O}-x_{0 w}$ averaged over the coordinates $70-90^{\circ} \mathrm{E}$ and $5-30^{\circ} \mathrm{N}$, from ERA40 reanalyses. Each point corresponds to a different month and year between Sept 1957 and Aug 2002. LHA was calculated from the surface precipitation rate, as described in the text. This relation
Steady states are defined by $\dot{x}=0$. Solving this condition together with the bifurcation condition $\partial F(x) / \partial x=0$ gives bifurcating steady states. This will be applied to our model. Whether or not a bifurcation is present depends on the value of $\partial F(x) / \partial x$, so contributions to this quantity are important. That is the motivation for studying the behavior of $\partial S_{\text {net }}^{f} / \partial x$ in Sect. 2.2.

\section{Data used and parameter estimates for July-mean Indian summer monsoon}

\subsection{Land and ocean regions for calculating parameters}

Parameters for the Indian summer monsoon (ISM) were estimated from ERA40 reanalyses, which includes the period between Sept 1957 and Aug 2002. Land-region parameters in our model are estimated by averaging over coordinate ranges $70-90^{\circ} \mathrm{E}$ and $5-30^{\circ} \mathrm{N}$. Tropospheric mean temperatures for the land-region are calculated over this same domain, averaging vertically from the surface to the pressure level of 100 mbar. Estimation of parameters for the ocean boundary conditions is confined to the Arabian sea, over $65-78^{\circ} \mathrm{E}$ and $5-30^{\circ} \mathrm{N}$, following Levermann et al. (2009). There is partial overlap in coordinates for the land and ocean regions. The model of horizontal winds for the Indian summer monsoon uses the same ocean region, i.e. $65-78^{\circ} \mathrm{E}$ and $5-30^{\circ} \mathrm{N}$. The zonal wind is computed over this region and used to estimate the parameter of Eq. (1), following the domain used for these calculations in Levermann et al. (2009).

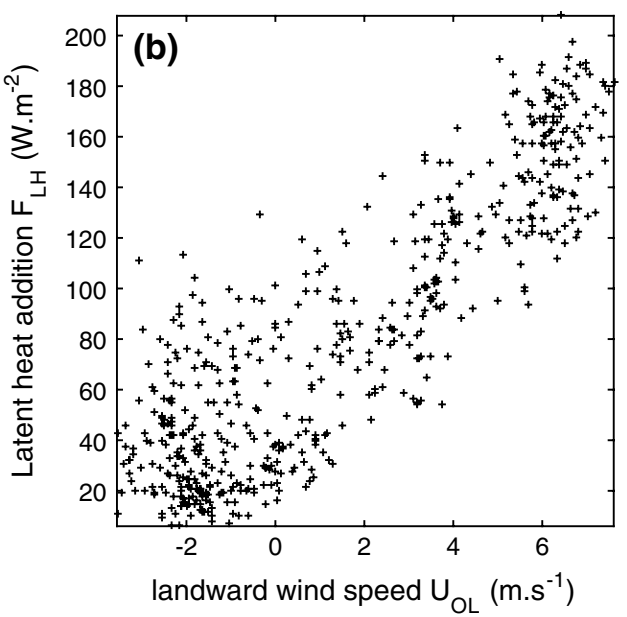

was used in the parameterization of Eq. (18) for LHA. The dashed line shows the results of nonlinear least-squares regression. The errors were added to the mean trend to obtain the following model: $F_{L H}=6.02 \times 10^{3}\left(\frac{\bar{T}_{L}-\bar{T}_{O}-x_{m}}{\bar{T}_{O}}\right)^{1.01}+\varepsilon_{F L H}$, with $\varepsilon_{F L H}$ being approximately Gaussian with standard deviation $27.7 \mathrm{~W} \mathrm{~m}^{-2}$; b graph of LHA versus landward wind-speed, with the latter indicated in Fig. 3 


\subsection{Parameterization of latent heat addition and wind-speed}

The term for LHA in the model was estimated from the average surface precipitation rate within this region, after weighing by area. This is plotted in Fig. 2. Multiplying the precipitation rate in $\mathrm{m} \mathrm{s}^{-1}$ by $\rho_{w} L$, where $\rho_{w}$ is water density and $L$ is latent heat capacity, yields the estimate for $F_{L H}$ in $\mathrm{W} \mathrm{m}^{-2}$, as shown in Fig. 2. In Fig. 2a the horizontal axis describes tropospheric mean temperature differences between land and ocean, $\bar{T}_{L}-\bar{T}_{O}$, minus $x_{0 w}$ at which surface wind reverses sign. Recall that the variable $\bar{T}_{L}-\bar{T}_{O}-x_{0 w}$ is simply $x$, the state variable in the governing equation. Each point is for a different month and year and the dashed line shows the mean trend obtained via nonlinear regression. The relationship is approximately linear. Figure $2 \mathrm{~b}$ is similar except with horizontal axis indicating landward wind-speed. Because of the close relation between wind-speed and temperature difference, either parameterization could have been used. In this paper we have chosen to represent LHA in terms of the variable related to temperature difference, i.e. $x$.

Figure 3 shows the zonal wind-speed calculated over the Arabian sea within $65-78^{\circ} \mathrm{E}$ and $5-30^{\circ} \mathrm{N}$, used in the parameterization to calculate landward wind-speed and its relation with temperature-difference. The winds are averaged over that depth of the lower troposphere through which winds have the same sign as at the surface. Hence

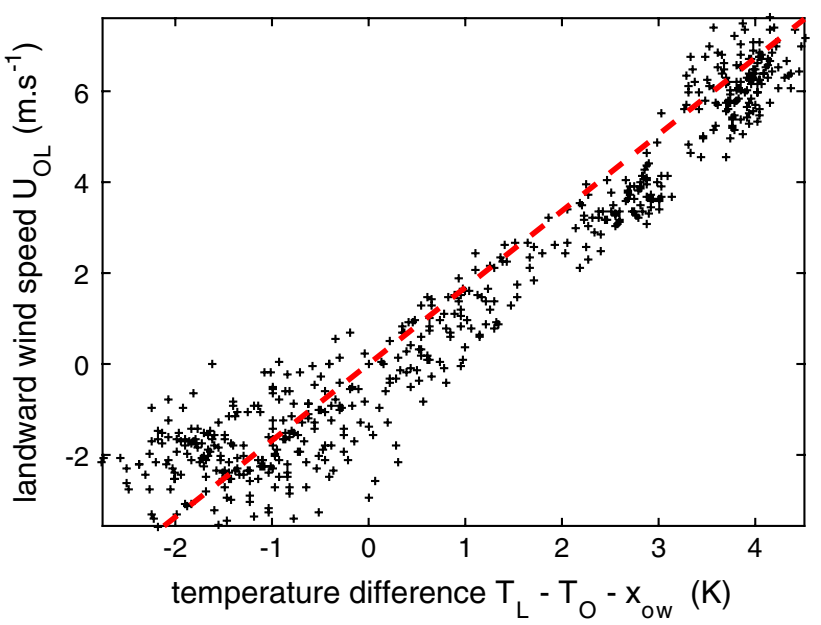

Fig. 3 Graph of landward wind-speed versus temperature difference $x \equiv \bar{T}_{L}-\bar{T}_{O}-x_{0 w}$. Each point shows ERA40 mean for a different month and year between Sept 1957 and Aug 2002. Dashed line shows the linear trend, following the parameterization in Eq. (1). The resulting model is $U_{O L}=1.68\left(\bar{T}_{L}-\bar{T}_{O}-x_{0 w}\right)+\varepsilon_{W O L}$, or equivalently $U_{O L}=1.68 x+\varepsilon_{W O L}$, with $\varepsilon_{W O L}$ being approximately Gaussian with standard deviation $0.95 \mathrm{~m} \mathrm{~s}^{-1}$. These errors are not stationary in $x$ as is clear from the graph. Despite this limitation the linear model of Eq. (1) is used for simplicity this depth varies with time. The landward wind-speed is plotted against state variable $x \equiv \bar{T}_{L}-\bar{T}_{O}-x_{0 w}$. The value of $x_{0 w}=-5.8 \mathrm{~K}$, and indicates the temperature difference where surface winds in this parameterization reverse sign. Therefore when $x=0$ the wind-speed $U_{O L}=0$ and the sign of $U_{O L}$ in the parameterization of Eq. (1) corresponds to the sign of $x$. That zero wind-speed occurs at negative land-ocean temperature contrast is simply a consequence of much of the land-surface lying significantly above sea level. When temperatures are averaged over the depth of the troposphere the corresponding pressure domains are different for the land and ocean regions. Therefore the precise value of $x_{0 w}$ does not carry physical significance.

\subsection{July-mean monsoon parameters}

For the parameters including lapse rate, surface pressures and tropospheric-mean temperatures, July-mean conditions were used, in order to analyze bifurcation behavior of the July-mean steady-state over India. The lapse-rate is calculated by least-squares regression of temperature versus geopotential height. This analysis is carried out for each combination of latitude-longitude coordinates, following which area-weighted averages are calculated separately for land and ocean. The available ERA40 data for July included the period 1958-2002. Similarly surface pressure was calculated for the land and ocean regions, by averaging over the domains defined in Sect. 3.1.

Table 1 shows parameter estimates for the model, including those made in Sects. 3.2. Where confidence intervals are present they denote uncertainties of the coefficient estimates, and not the estimates of the stochastic error present in the parameterization, such as in Figs. 2

Table 1 Parameter estimates and uncertainty (for July-mean conditions unless otherwise specified) within the Indian summer monsoon

\begin{tabular}{ll}
\hline Parameter & Estimate \\
\hline $\bar{T}_{O}$ & $268.8 \pm 0.6 \mathrm{~K}$ \\
$\Gamma_{O}$ & $6.39 \times 10^{-3} \pm 1.01 \times 10^{-4} \mathrm{~K} \mathrm{~m}^{-1}$ \\
$\Gamma_{L}$ & $6.37 \times 10^{-3} \pm 1.04 \times 10^{-4} \mathrm{~K} \mathrm{~m}^{-1}$ \\
$p_{0 O}$ & $970 \mathrm{mbar}$ \\
$p_{0 L}$ & $880 \mathrm{mbar}$ \\
$x_{0 w}$ & $-5.84 \mathrm{~K}$ \\
$x_{m}$ & $-8.59 \mathrm{~K}$ \\
$k_{w}$ & $1.68 \pm 0.056 \mathrm{~m} \mathrm{~s}^{-1} \mathrm{~K}^{-1}$ \\
$k_{L H}$ & $6020 \pm 1966 \mathrm{~W} \mathrm{~m}^{-2}$ \\
$\alpha_{L H}$ & $1.01 \pm 0.084$ \\
$A$ & $3.2 \times 10^{12} \mathrm{~m}^{2}$ \\
$B$ & $9.8 \times 10^{6} \mathrm{~m}$ \\
\hline
\end{tabular}

Error bars indicate $95 \%$ confidence intervals 


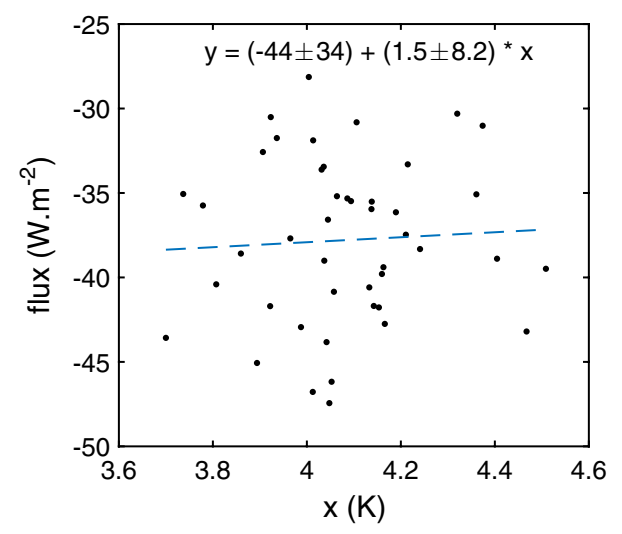

Fig. 4 Sum of surface sensible heat flux $F_{B}^{S}$, net shortwave flux $F_{n e t}^{S W}$, and net longwave flux $F_{N e t}^{L W}$ into the control volume versus state variable $x$ from ERA40 reanalysis averaged for July for the Indian summer monsoon land region between $70-90^{\circ} \mathrm{E}$ and $5-30^{\circ} \mathrm{N}$. Each point corresponds to a different year between 1958 and 2002. Also shown is the linear regression line, its equation, and $95 \%$ confidence intervals for coefficients. The $95 \%$ confidence interval of the ordinate in this figure is $-37.8 \pm 9.68 \mathrm{~W} \mathrm{~m}^{-2}$

and 3. We reiterate that, for the two parameterizations in the model, involving surface wind-speed in Eq. (1) and LHA in Eq. (18), we fit models based on ERA40 data for all months that are available, because these variables have simple relations with the state variable and therefore their behavior near an arbitrary bifurcation point can be understood by characterizing their relationship over the entire annual cycle, which includes periods where state variable $x$ approaches zero. By contrast it is not clear whether the behavior of lapse rates and surface pressure can be generalized across the year via a simple relationship and therefore, for convenience, we confine their estimation to Julymean conditions when the monsoon is strongest. This latter restriction limits the interpretation that can be made from the numerical results that follow, because many estimates are based on July-mean conditions alone.

\subsection{Sum of sensible, shortwave and longwave heat fluxes for July monsoon conditions}

There are many contributions to the balance of DSE in the governing equation, but a simplification can be made for analyzing bifurcation of this model, because the combined effect of some terms becomes independent of the state variable. Figure 4 shows the sum of sensible heat flux, and the net shortwave and longwave heat flux convergence, versus the state variable from ERA40 reanalyses for July conditions. Averages have been calculated between $70-90^{\circ} \mathrm{E}$ and $5-30^{\circ} \mathrm{N}$. Also shown is the least-squares regression line together with its equation. The slope of the linear regression does not differ significantly from zero, and we therefore assume that the sum of these fluxes does not vary systematically with the variable $x$, and therefore with the land-ocean temperature difference, during the month of July. For the Indian summer monsoon in July, the contributions to the balance of DSE that depend systematically on the land-ocean temperature difference are therefore only the horizontal flux of DSE and the LHA to the control volume. Hence we shall only consider these two contributions to the energy balance in the next section when we examine the model's bifurcation behavior.

\section{Results}

\subsection{Simplification to the model of dry static energy flux}

The model of DSE flux is simplified by recognizing that low-level convergence is on average confined to layers near the surface so that $\frac{p_{0 O}-p_{D}}{p_{0 O}} \ll 1$. Then $\ln \frac{p_{0 O}}{p_{D}}=$ $\ln \frac{p_{0 O}-p_{D}+p_{D}}{p_{D}} \cong \frac{p_{0 O}-p_{D}}{p_{D}}$ using the relation $\ln (1+x) \cong x$ for small $x$, and likewise $\ln \frac{p_{0 L}}{p_{D}} \cong \frac{p_{0 L}-p_{D}}{p_{D}}$. Using the relations $\quad-x_{0 w} / \bar{T}_{O} \ll 1, \quad f_{D O}=\frac{p_{D}}{p_{0 O}-p_{D}} \gg 1, \quad$ and $k_{O} \sim k_{L} \sim \frac{9.8-6}{9.8} \cong 0.4$, the value of the coefficient $c_{01}$ appearing in Eq. (19) simplifies to $c_{01} \cong \frac{R}{c_{p}} \frac{\Gamma_{D}-\Gamma_{O}}{\Gamma_{D}} \bar{T}_{O}$ and similarly $c_{11} \cong 1+\frac{R}{c_{p}}$. Then the flux under monsoonal circulations while $x>0$ is approximately

$S_{n e t}^{f} \cong-\frac{B k_{w}}{\Gamma_{D}}\left(p_{0 O}-p_{D}\right)\left\{\left(\frac{R}{c_{p}} \frac{\Gamma_{D}-\Gamma_{O}}{\Gamma_{D}} \bar{T}_{O}\right) x+\left(1+\frac{R}{c_{p}}\right) x^{2}\right\}$

where we have substituted $g / c_{p}=\Gamma_{D}$. In case of reversed circulations we make analogous simplifications for approximation

$S_{n e t}^{f} \cong \frac{B k_{w}}{\Gamma_{D}}\left(p_{0 L}-p_{D}\right)\left\{-\left(\frac{R}{c_{p}} \frac{\Gamma_{D}-\Gamma_{L}}{\Gamma_{D}} \bar{T}_{O}\right) x+\left(1+\frac{\Gamma_{L}}{\Gamma_{D}} \frac{R}{c_{p}}\right) x^{2}\right\}$

The terms that are linear in $x$ originate in the respective lapse rates being smaller than dry-adiabatic. The magnitudes of corresponding linear coefficients increase with departures from dry-adiabatic conditions, and also with the weight of the converging layer and sensitivity of winds according to $k_{w}\left(p_{0 O}-p_{D}\right)$ and $k_{w}\left(p_{0 L}-p_{D}\right)$ in the cases of monsoonal and reversed circulations respectively. The interpretation of these linear terms is that they arise from vertical gradients in DSE, in contrast to the quadratic terms arising from horizontal gradients. In previous simplified models (Levermann et al. 2009; Schewe et al. 2011) of monsoon bifurcations the vertical variation in temperature was not treated explicitly and hence only the quadratic term from horizontal gradients arose. 
Let us consider the relative role of these two contributions. Because $R / c_{p} \cong 1 / 3$ for dry air, the ratio of linear and quadratic terms is approximately $\bar{T}_{O} / 10 x$. This is much larger than 1 and the linear term is dominant. Horizontal fluxes of DSE are governed by vertical gradients of this quantity, contrary to previous models where horizontal gradients play the only role (Levermann et al. 2009; Schewe et al. 2011). This affects nonlinear dynamics, as will be shown.

\subsection{Condition for stable monsoon solution}

For our simplified one-dimensional governing equation to be plausible it must at least admit a stable steady-state monsoon. Let $x_{*}$ be a steady-state solution to the governing Eq. (17) under fixed external conditions. For it to be stable the eigenvalue should be negative, or equivalently

$G_{T} \lambda\left(x_{*}\right)=\frac{\partial F\left(x_{*}\right)}{\partial x}=\frac{\partial F_{L H}\left(x_{*}\right)}{\partial x}+\frac{1}{A} \frac{\partial S_{n e t}^{f}\left(x_{*}\right)}{\partial x}<0$

where we have used the observation that the other contributions together can be treated as independent of $x$ (Sect. 3.4). Considering the regime where $x>0$ and applying Eqs. (18) and (22) and making the approximation that $\alpha_{L H}=1$ (Fig. 2; Table 1) the condition is

$$
\begin{aligned}
G_{T} \lambda\left(x_{*}\right)= & \frac{k_{L H}}{\bar{T}_{O}}-\frac{B k_{w}}{A \Gamma_{D}}\left(p_{0 O}-p_{D}\right) \\
& \times\left\{\frac{R}{c_{p}} \frac{\Gamma_{D}-\Gamma_{O}}{\Gamma_{D}} \bar{T}_{O}+2\left(1+\frac{R}{c_{p}}\right) x_{*}\right\}<0
\end{aligned}
$$

so that the sensitivity of the DSE flux to the temperature difference must be sufficiently large, when compared to $k_{L H} / \bar{T}_{O}$.

Parameters in the above model have been estimated from ERA40 reanalysis, except pressure $p_{D}$ at which overturning occurs. This is not estimated directly because the model's geometry is idealized, and doesn't accommodate complexities of real monsoonal circulations in which, for example, winds are not landward everywhere. Whether or not such a model can reproduce actual behavior of the DSE flux depends on whether it ab-initio represents correctly the relation between linear and quadratic terms involved in describing $S_{n e t}^{f}$. Without directly estimating the DSE flux from reanalysis data, we cannot determine if this occurs. However our simplification of the DSE flux in Sect. 4.1 indicates that this is likely to be the case, because the relation between linear and quadratic terms is straightforward and is governed by the ratio of vertical and horizontal gradients in DSE.

We therefore treat $p_{D}$ as a tuning parameter, estimating its value so that the model's steady state approximates

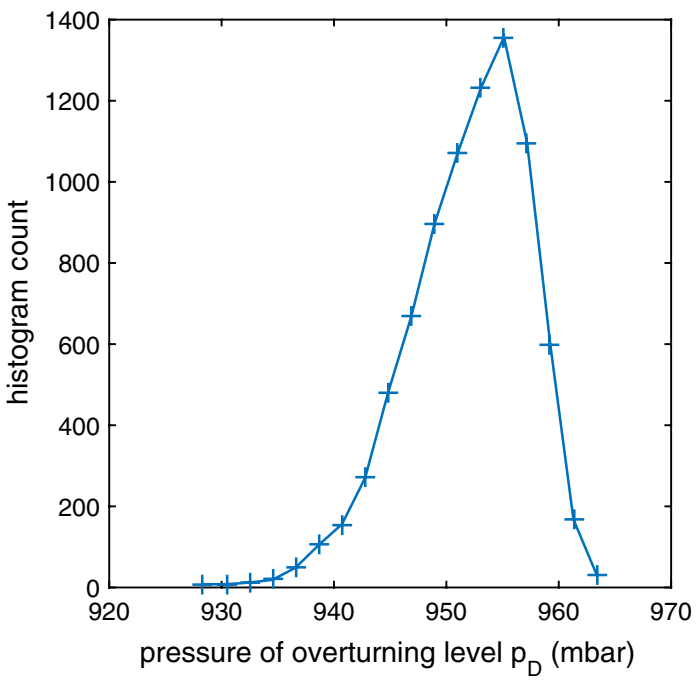

Fig. 5 Histogram counts of overturning level $p_{D}$ estimated by tuning its value in the model to simulate steady state $x_{*}$ sampled from the latter's distribution in ERA40 under July conditions. The mean value of $p_{D}$ is 952 mbar and mode occurs around 955 mbar. Its distribution is confined to near-surface pressures. Only simulations with a resulting stable steady-state, numbering 8229 out of 10,000 simulations made, have been used in these calculations

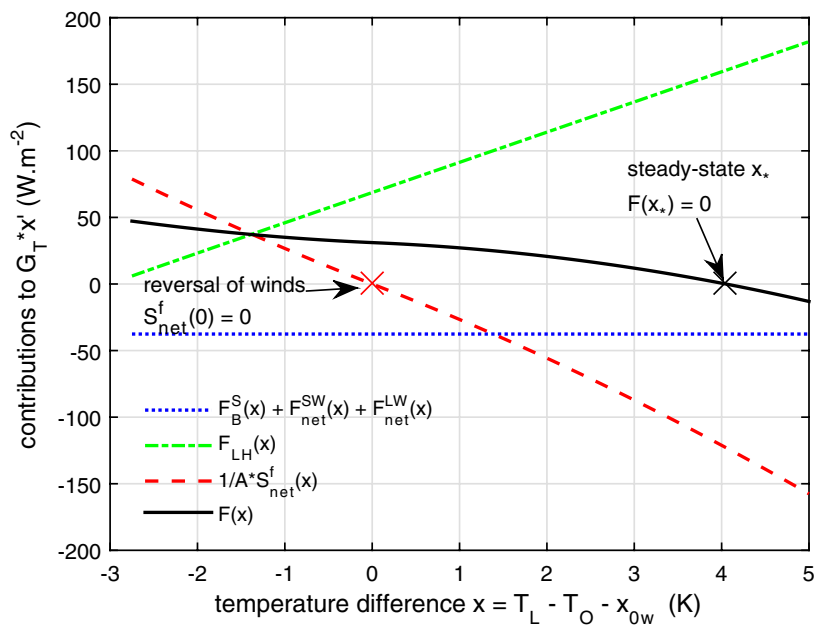

Fig. 6 Contributions to the measure of warming rate in the model, i.e. $G_{T}(x) d x / d t$, versus $x$. Each curve shows corresponding sample mean for that process of the Monte-Carlo simulations of the model. Black curve shows the total contribution $F(x)$. Steady-state is where the black curve crosses the abscissa, or $F(x)=0$. The dashed red curve, the DSE flux, is only weakly nonlinear. This is due to the dominant role of its linear contribution, arising from vertical gradients in DSE. Hence this term has nonzero slope at the origin, where $S_{\text {net }}^{f}=0$, so that significantly $\partial S_{\text {net }}^{f} / \partial x \neq 0$. There is no bifurcation in these mean results, because nowhere is $\partial F / \partial x=0$. For these numerical simulations we have not estimated pressure depth $p_{0 L}-p_{D}$ of the diverging layer in case of reversed circulation in the model, but instead assumed it to be equal to pressure depth $p_{0 O}-p_{D}$ of the converging layer during monsoonal circulations 
corresponding values in ERA40 reanalyses. The reanalysis steady-state is simulated from a Gaussian distribution with $95 \%$ confidence interval $x_{*}=4.08 \pm 0.36 \mathrm{~K}$, corresponding to the distribution in ERA40 of the state variable during July months. Following Monte-Carlo simulation that considers uncertainties presented here, including errors in estimation of the model of winds and LHA, for each realization the value of $p_{D}$ is chosen to minimize the difference between model $x_{*}$ and the corresponding sample from its distribution. What follows is a distribution of overturning level $p_{D}$, graphed in Fig. 5. Only cases with stable steady-state, corresponding to where the condition in Eq. (25) is satisfied, are included. The sample mean of $p_{D}$ is 952 mbar and the distribution of overturning pressure is confined to values near the surface. The control volume experiences continuous inflow from all sides of its boundary, whereas in actual monsoon systems the inflow is confined to a small fraction of the boundary. Hence our model has an artificially thin inflow layer. The mean depth of the inflow layer in the model is only $p_{0 O}-p_{D}=970-952=18$ mbar.

The direction of the circulation is governed by the sign of $x$ and not the thickness of the inflow layer, which is defined to be positive. The above value of $p_{D}$ is estimated for July-mean conditions, when low-level flow is landward. For bifurcation behavior we are only concerned with the regime where circulation is monsoonal. We have not estimated the value of the overturning level as a function of $x$. Therefore the above estimate is not valid where circulation is reversed, corresponding to where low-level flow is oceanward. We recall that for reversed circulation the DSE flux depends on pressuredepth $p_{0 L}-p_{D}$ over land instead of the pressure depth over ocean. For our numerical simulations below we do not estimate this pressure depth $p_{0 L}-p_{D}$ for the reversed circulation in our model, but instead assume that it is equal to the pressure depth $p_{0 O}-p_{D}$ during monsoonal circulations.

Figure 6 shows mean contributions to the rate of change of temperature from that Monte-Carlo simulation. The mean $x_{*}$ thus obtained closely approximates the mean of the aforementioned distribution from ERA40. The DSE flux is only weakly nonlinear, reflecting the dominant role of the linear term as described in Sect. 4.1. Near the steadystate, cooling by DSE flux balances a significant part of heating by LHA. There is no bifurcation in the model resulting from these mean tendencies, specifically nowhere is $\partial F / \partial x$ equal to zero. We consider bifurcation behavior more closely in the next section. Here we observe that, due to the linear contribution, the slope of the flux term cannot be neglected at $x=0$ (Fig. 6).

\subsection{Condition for a bifurcation point}

If the steady state at $x=x_{b}>0$, occurring for some value of shortwave forcing, bifurcates then the corresponding eigenvalue vanishes, i.e. $\lambda\left(x_{b}\right)=0$. As discussed previously the only nonzero contributions to this are $\frac{\partial F_{L H}(x)}{\partial x}$ and $\frac{1}{A} \frac{\partial S_{n e t}^{f}(x)}{\partial x}$. The first term is positive and approximately constant, being equal to $k_{L H} / \bar{T}_{O}$. The second term is negative

(a)

This model

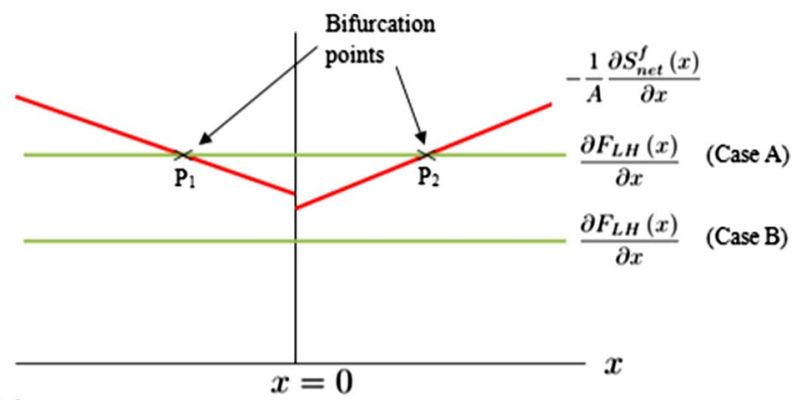

(b)

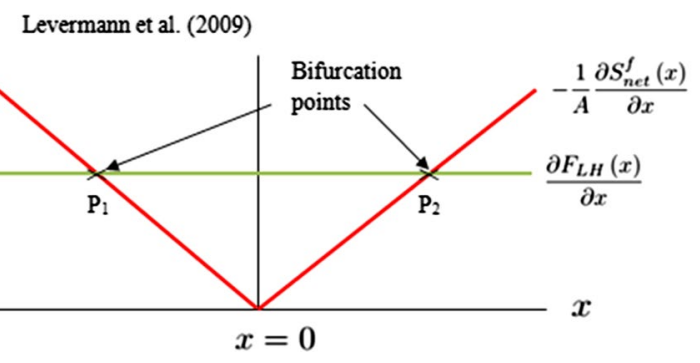

Fig. 7 Illustration of the condition for bifurcation, involving contributions to the value of $\partial F(x) / \partial x$ in Eq. (17): a the model presented here with dry static energy flux containing a term linear in land-ocean temperature difference, due to vertical gradients, and a term that is quadratic, due to horizontal gradients. Therefore $-\frac{1}{A} \frac{\partial S_{n e t}^{f}(x)}{\partial x}$ is linear in $x$ with nonzero intercept at $x=0$. Latent heat addition is idealized as being linear in temperature difference, so $\frac{\partial F_{L H}(x)}{\partial x}$ is constant. Stability requires that for sufficiently large $x$ the magnitude of $-\frac{1}{A} \frac{\partial S_{n e t}^{f}(x)}{\partial x}$ is larger than that of $\frac{\partial F_{L H}(x)}{\partial x}$ Stability exists to the left of point $\mathrm{P}_{1}$ and right of point $\mathrm{P}_{2}$ in case $\mathrm{A}$, and everywhere for case $\mathrm{B}$. Bifurcation points correspond to intersections between the curves of $\frac{\partial F_{L H}(x)}{\partial x}$ and $-\frac{1}{A} \frac{\partial S_{n e t}^{f}(x)}{\partial x}$. Bifurcation is not inherent to this model. There are two bifurcation points $\mathrm{P}_{1}$ and $\mathrm{P}_{2}$ in case $\mathrm{A}$ and none in case $\mathrm{B}$. From the graph a necessary condition for bifurcation is that $\frac{\partial F_{L H}(x)}{\partial x}$ is larger than the minimum value of $-\frac{1}{A} \frac{\partial S_{n e t}^{f}(x)}{\partial x}$, which occurs at $x=0$; $\mathbf{b}$ origin of bifurcation in the model of Levermann et al. (2009). There, DSE flux does not contain a linear term, so that the intercept of $-\frac{1}{A} \frac{\partial S_{n e t}^{f}(x)}{\partial x}$ is zero. Then, because the magnitude of $-\frac{1}{A} \frac{\partial S_{\text {net }}^{f}(x)}{\partial x}$ must be larger than that of $\frac{\partial F_{L H}(x)}{\partial x}$ for sufficiently large $x$, in order for the present steady-state to be stable, there must be an intersection between the two curves for some value of $x>0$ and hence a bifurcation point is inherent to such a model 
and, $S_{\text {net }}^{f}(x)$ being quadratic in $x$, the magnitude of $\frac{1}{A} \frac{\partial S_{\text {net }}^{f}(x)}{\partial x}$ grows linearly with $x$. As described in Sect. 4.2 the condition for a stable steady-state under present conditions is that the magnitude of $\frac{1}{A} \frac{\partial S_{n e t}^{f}(x)}{\partial x}$ eventually becomes larger than $k_{L H} / \bar{T}_{O}$; specifically this relation must occur at present steady-state $x_{*}$. Geometrically it is seen that a necessary condition for some bifurcation point $0<x_{b}<x_{*}$ to exist is that $k_{L H} / \bar{T}_{O}>-\frac{1}{A} \frac{\partial S_{n e t}^{f}(0)}{\partial x}$ or

$\frac{k_{L H}}{\bar{T}_{O}}-\frac{B k_{w}}{A \Gamma_{D}}\left(p_{0 O}-p_{D}\right) \frac{R}{c_{p}} \frac{\Gamma_{D}-\Gamma_{O}}{\Gamma_{D}} \bar{T}_{O}>0$

as illustrated in Fig. 7a. This panel shows the origin of a bifurcation point, if it exists, in the model presented here. Two cases are shown, with and without bifurcation. Bifurcation is not inherent to this model and depends on the relation between the sensitivities to temperature difference of DSE flux and LHA. By contrast the model of Levermann et al. (2009) has bifurcation inherent to it, regardless of model parameters, as shown in Fig. 7b. That model's contribution from the flux consists only of a quadratic term in $x$, so that intersection between the curves of $-\frac{1}{A} \frac{\partial S_{\text {net }}^{f}(x)}{\partial x}$ and $\frac{\partial F_{L H}(x)}{\partial x}$ necessarily occurs at some value of $x>0$ and a bifurcation occurs there, corresponding to a tipping point in the model monsoon.

Let us examine if a bifurcation occurs for our model, or equivalently whether it is similar to case A or case B in Fig. 7a. Figure 8a plots contributions to the value of $\partial F(x) / \partial x$, following the schematic shown in Fig. 7a. Mean values and uncertainty ranges are graphed for the contributions of LHA and the negative of the flux's contribution. Recall that if these curves intersect the eigenvalue is zero, indicating bifurcation. The mean curves do not intersect, indicating no bifurcation in this case. This is consistent with the mean results in Fig. 5, where bifurcation is absent and $G_{T}(x) d x / d t$ is nowhere zero.

However the uncertainty ranges do overlap in Fig. 8a, suggesting that the above model can admit bifurcations consistent with reanalysis data. Figure $8 \mathrm{~b}$ plots histogram counts of the value of Eq. (26). We recall from earlier in this section that positive values of this quantity indicate that a bifurcation is present in the model, because the two curves must intersect, as in case A of Fig. 7a. Bifurcation occurs in the model under sampling of its parameter values in approximately $32 \%$ of the cases, and this is our estimate of the probability that the inequality (26) is satisfied. The possibility of case A with bifurcations should be considered, although it appears much less probable than not.

We now consider the differences between the cases with and without bifurcations. Figure 9 plots the mean of cases without and with bifurcations respectively, in left and right panels. The behavior of LHA is assumed to be linear. Upper panels show contributions to the value of $\partial F(x) / \partial x$, so where the two graphs intersect a bifurcation occurs. Lower panels show contributions to the measure of warming rate. The black curve's crossing of the abscissa where $F(x)=0$ indicates the steady-state. Where the curve for $F(x)$ has zero slope, there is a bifurcation point. Contrasting these two sets of graphs shows that bifurcation behavior in the right panel corresponds mainly to reduced sensitivity of the DSE flux, and this is compensated by slightly reduced sensitivity of LHA. As a result the steady-state is the same in the two cases, but the graphs for DSE flux and

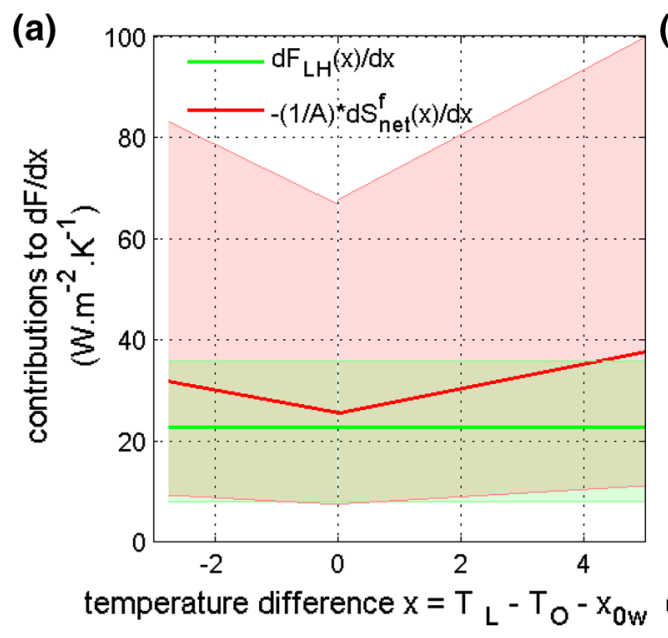

Fig. 8 Contributions to the value of $\partial F(x) / \partial x$ and illustration of the necessary condition for a bifurcation in the model presented here: a contributions $\frac{\partial F_{L H}(x)}{\partial x}$ and $-\frac{1}{A} \frac{\partial S_{n e t}^{\prime}(x)}{\partial x}$ versus temperature-difference $x$. Where these two curves intersect a bifurcation occurs. Shading indicates corresponding uncertainty ranges obtained via Monte Carlo

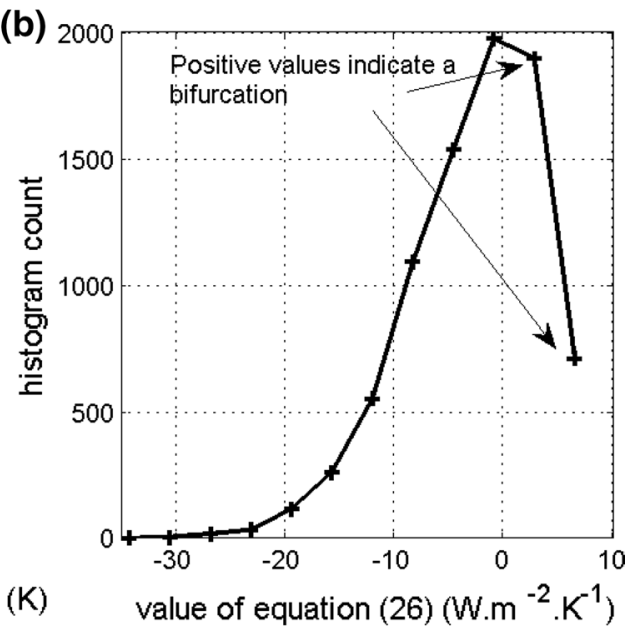

simulation as described in Sect. 4.2. The mean curves do not intersect but the uncertainty ranges overlap; $\mathbf{b}$ histogram counts of the value of Eq. (26), whose importance is that positive values correspond to the necessary condition for a bifurcation. Positive values occur in $32 \%$ of the samples 
(a)

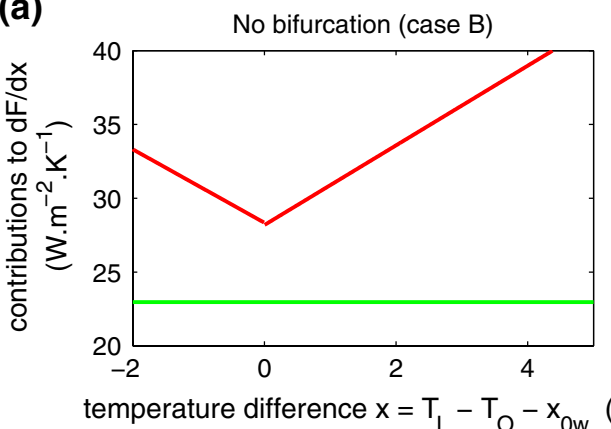

(c)

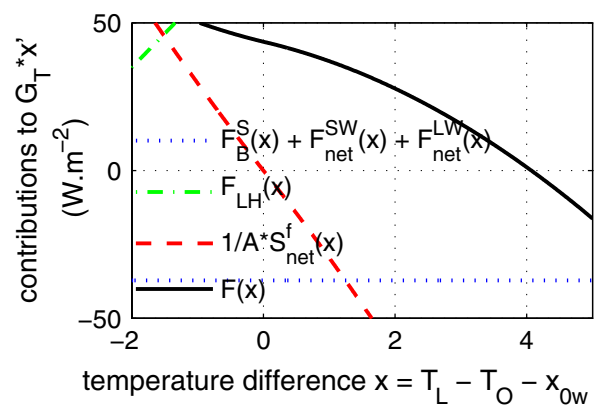

(b)

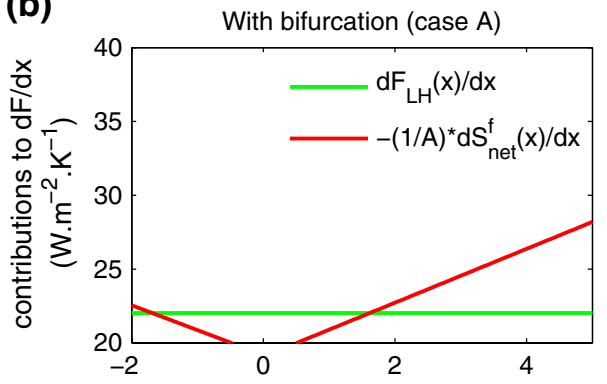

(K)

(d)

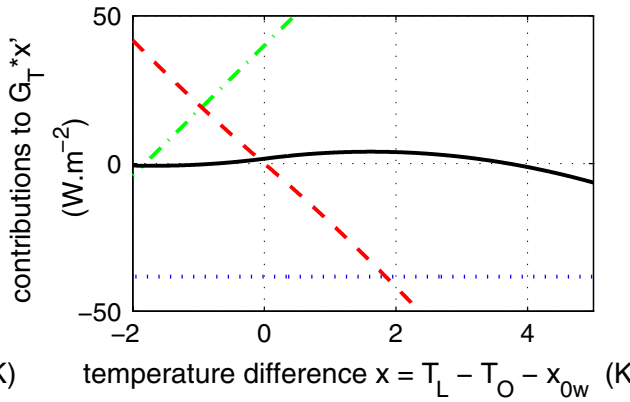

(K)

c mean of cases with no bifurcation; d corresponding mean of cases with two bifurcation points. Locations with zero slope in the black curve are bifurcation points, corresponding to intersections between the graphs in b. Bifurcation behavior corresponds in the model to lower sensitivity of DSE flux to land-ocean temperature-contrast combined with slightly lower sensitivity of LHA, so that the upperpanel's variables can intersect, whereas the steady-state in the two cases is approximately the same

intersection with the curve for LHA becomes possible, as in Fig. 9b. Bifurcation occurs more easily in the model if the inflow layer is relatively thin or winds are less sensitive to the temperature-contrast.

As far as the model introduced in this paper is concerned, both cases A and B in Fig. 9 are physically realistic. They both correspond to monsoon-type circulations characterized by their DSE balance. However there are also striking differences between the cases. The case with a bifurcation has a much lower sensitivity of $F(x)$ to $x$. $F(x)$ characterizes contributions to the DSE balance. Therefore by introducing additional empirical constraints one might further limit possibilities for the model's nonlinear dynamics.

It is not necessary that the nonlinear contribution to the DSE flux have an essential role in the occurrence of bifurcation. For example if the LHA had been nonlinear in the value of $x$ then an intersection different from the ones in Figs. $7 \mathrm{a}$ and $9 \mathrm{~b}$, but still corresponding to bifurcation, would become possible even in the hypothetical case where the nonlinearity of the DSE flux is negligible. However $k_{w}\left(p_{0 O}-p_{D}\right)$ in our model. Where this factor is smaller, an 
the quadratic term in the DSE flux represents a real phenomenon, the effect of horizontal gradients in DSE on the changes in the energy balance due to horizontal winds, even though this term's magnitude is generally small. Therefore we conclude that horizontal gradients in DSE are important to potential bifurcations in our model, despite these gradients being smaller than vertical gradients. The effect of vertical gradients in the DSE is to make the presence of bifurcations uncertain despite the above nonlinearity, and also make further details about the model germane to this question.

\section{Discussion}

The box-model of dry static energy balance presented in this paper includes many idealizations. An important simplification involves the overall scheme of overturning and winds underlying treatment of the dry static energy (DSE) flux. Therefore these results are mainly indicative, suggesting how bifurcations can arise in minimal monsoon models. Despite its simplicity, the model furnishes an account consistent with stable steady-state solutions.

The model shows that nonlinear DSE flux does not necessarily lead to a bifurcation, contradicting results of prior reduced models (Levermann et al. 2009; Schewe et al. 2011). The difference here is because of the nonzero sensitivity to land-ocean temperature difference of the DSE flux, at the limit where the wind-speed vanishes. Such behavior arises from the inclusion of a linear term in the model of the DSE flux. This linear term is due to the vertical lapse rate of temperature being smaller than the dry adiabatic value. Then the DSE increases strongly with height, causing small horizontal winds to produce proportional changes in the DSE balance in circulations where the control volume's mass is approximately conserved. In addition to this linear term, there is a quadratic term analogous to these previous models (Levermann et al. 2009; Schewe et al. 2011), but this quadratic term is comparatively small.

This paper has not estimated the DSE flux directly from reanalyses. However, the model of DSE flux admits the simplification introduced in Sect. 4.1, which is easy to explain and interpret. Therefore the account of the bifurcation that follows might be relevant beyond this particular model. Specifically, this work suggests that box-models of DSE flux should contain terms that are both linear and quadratic in the horizontal temperature-difference, or alternately in measures of wind-speed. These originate respectively in vertical and horizontal gradients of the DSE. Furthermore, combining the equations for DSE flux with the other aspects of the DSE balance can lead to equations of higher-degree in the state variable, as previous authors
(Levermann et al. 2009; Boos and Storelvmo 2016) have shown. One effect of the simplifications made here is that certain details of the model, such as the horizontal temperature-difference at which surface-wind reverses direction, are not important for its nonlinear dynamics.

Another limitation of the model is its restriction to a single ordinary differential equation. As the scatter in Fig. 2 indicates, latent heat addition (LHA) is not only a function of $x$, and higher-dimensional models might therefore be required to represent the essential dynamics. The errors in estimating these models of surface-winds and the LHA have been treated as being stationary, and therefore not included in calculations of the contributions to the variable $\partial F / \partial x$ that governs the model's bifurcation behavior. More complete higher-dimensional models, for example including prognostic column water, might have different nonlinear dynamics. We have considered one-dimensional models in order to elaborate and engage with issues raised by previous one-dimensional models of monsoon transitions cited here. Such a minimalistic approach might be evolved towards understanding and developing higher-dimensional models, by identifying candidate processes that might combine to produce a bifurcation in more complex models.

The main lesson from this study is that bifurcation is not inherent to monsoon models. Specifically, it is not necessary that $\partial F / \partial x$ must be zero anywhere in Eq. (17). This is because of the strong vertical gradients in DSE, induced by the dry thermal stratification of the atmosphere. In this respect our conclusion is similar to that of Boos and Storelvmo (2016), who find that accounting for this effect eliminates the bifurcation in their model.

However in contrast with Boos and Storelvmo (2016), our results do admit the possibility of a bifurcation. Since the larger sensitivity of DSE flux to temperature differences is essential for stabilizing monsoons, the LHA must be more sensitive in some intermediate regime for these processes' respective curves to cross in graphs analogous to Fig. 7a and for a bifurcation to occur. In case LHA depends linearly on the land-ocean temperature difference, as it approximately does for the parameterizations estimated here, the sufficient condition for bifurcation is quite straightforward and noted in Eq. (26) as well as illustrated in Fig. 7a. In the more general case, as for other monsoon regions, or for alternate parameterizations, the relationship for LHA might be nonlinear. Even here sufficient conditions for a bifurcation are easily deduced. Consider the average value of $\partial F_{L H}(x) / \partial x$ over $x$, i.e. the mean rate of increase of this flux with $x$. If this quantity is larger than $-(1 / A) \partial S_{\text {net }}^{f}(x) / \partial x$ at $x \rightarrow 0^{+}$, i.e. with monsoonal circulation at the limit of zero windspeed, a bifurcation point must exist. This is because, for some $x$, the value of $\partial F_{L H}(x) / \partial x$ must be larger than its average, and together these conditions would ensure intersection between the two curves in Fig. 7a. Hence if the 
mean sensitivity of LHA is larger than that of DSE flux when winds reverse, a bifurcation must occur in the model. It appears important to understand whether models that treat both moisture and temperature dynamics, and where moisture plays a more direct role in addition to its contribution to the DSE, can admit such behavior.

The version of the model presented here with the mean values of its parameters, in some sense the best-estimate model, does not exhibit bifurcation. There are significant uncertainties in estimating this model, as should be clear from Fig. 8. Furthermore some parameter combinations do admit bifurcations. However we have only considered a very limited number of observations to constrain this model, and including additional variables as constraints may further limit possibilities for its nonlinear dynamics. For example the two cases in Fig. 9, with and without bifurcation, differ significantly in their sensitivity of the DSE balance to the land-ocean temperature contrast.

This paper describes the range of behaviors possible and also characterizes differences between those cases with and without bifurcation. Smaller sensitivity of the DSE flux combined with slightly less sensitive LHA can create bifurcation, and hence it is important to characterize these processes more fully to understand what controls them.

Acknowledgments This research is supported by Divecha Centre for Climate Change at Indian Institute of Science. Thanks to Professor J Srinivasan for suggesting this topic, and to many colleagues for helpful discussion. Two reviewers made suggestions that substantially improved the paper.

\section{Appendix: Equation for tropospheric mean temperature}

It is convenient to describe vertical temperature variation in the model in terms of surface air temperature and mean lapse rate. However the state variable in the model is directly related to tropospheric mean temperature. Therefore this appendix describes the relation between these variables. Density-weighted tropospheric mean temperature is

$\bar{T}_{L}=\frac{\int_{0}^{z_{T}} \rho T_{L}(z) d z}{\int_{0}^{z_{T}} \rho d z}$

and using the hydrostatic relation $\rho d z=-(1 / g) d p$, the integral in the numerator becomes

$-\frac{1}{g} \int_{p_{0 L}}^{0} T_{L}(z) d p=-\frac{1}{g} \int_{p_{0 L}}^{0}\left(T_{L 0}-\Gamma_{L} H_{s L} \ln \frac{p_{0 L}}{p}\right) d p$

which simplifies to $\left(T_{L 0}-\Gamma_{L} H_{S L}\right) p_{0 L} / g$ with $H_{S L}=R \bar{T}_{L} / g$ being the scale height of pressure over land. The scale height is assumed to be independent of height and vary only with tropospheric mean temperature. The denominator in Eq. (27) simplifies to $p_{0 L} / g$ and therefore

$\bar{T}_{L}=T_{L 0}-\Gamma_{L} H_{s L}$

which is the relation used in the paper.

\section{References}

Agnihotri R, Dutta K, Bhushan R, Somayajulu B (2002) Evidence for solar forcing on the Indian monsoon during the last millennium. Earth Planet Sci Lett 198:521-527. doi:10.1016/ S0012-821X(02)00530-7

Boos WR, Storelvmo T (2016) Near-linear response of mean monsoon strength to a broad range of radiative forcings. Proc Nat Acad Sci USA 113:1510-1515. doi:10.1073/pnas.1517143113

Clemens S, Prell W, Murray D, Shimmield G, Weedon G (1991) Forcing mechanisms of the Indian Ocean monsoon. Nature 353:720 725. doi: $10.1038 / 353720 \mathrm{a} 0$

Crawford JD (1991) Introduction to bifurcation theory. Rev Mod Phys 63:991-1037. doi:10.1103/RevModPhys.63.991

Davis MB, Shaw RG (2001) Range shifts and adaptive responses to quarternary climate change. Science 292:673-679. doi:10.1126/ science.292.5517.673

Guckenheimer J, Holmes P (2002) Nonlinear oscillations, dynamical systems, and bifurcations of vector fields. Applied mathematical sciences (Book 42). Springer, Berlin

Iooss G, Joseph DD (1997) Elementary stability and bifurcation theory. Springer, Berlin

Knopf B, Flechsig M, Zickfeld K (2006) Multi-parameter uncertainty analysis of a bifurcation point. Nonlinear Process Geophys 13:531-540. doi:10.5194/npg-13-531-2006

Kutzbach JE, Guetter PJ (1984) Milankovitch and climate: understanding the response to astronomical forcing. In: Berger A, Imbrie J, Hays J, Kukla G, Saltzman B (eds) The sensitivity of monsoon climates to orbital parameter changes for 9000 Years BP: experiments with the NCAR general circulation model. Springer, Berlin, pp 801-820

Kutzbach JE, Liu Z (1997) Response of the African monsoon to orbital forcing and ocean feedbacks in the middle Holocene. Science 278:440-443. doi:10.1126/science.278.5337.440

Lenton TM, Held H, Kriegler E, Hall JW, Lucht W, Rahmstorf S, Schellnhuber HJ (2008) Tipping elements in the Earth's climate system. Proc Nat Acad Sci USA 105:1786-1793. doi:10.1073/ pnas.0705414105

Levermann A, Schewe J, Petoukhov V, Held H (2009) Basic mechanism for abrupt monsoon transitions. Proc Nat Acad Sci USA 106:20572-20577. doi:10.1073/pnas.0901414106

Li C, Yanai M (1996) The onset and interannual variability of the Asian summer monsoon in relation to land-sea thermal contrast. J Clim 9:358-375. doi:10.1175/1520-0442(1996)009<0358:TOA IVO $>2.0 . \mathrm{CO} ; 2$

Luo H, Yanai M (1983) The large-scale circulation and heat sources over the Tibetan Plateau and surrounding areas during the early summer of 1979, I. Precipitation and kinematic analyses. Mon Weather Rev 111:922-944. doi:10.1175/1520-0493(1983)111<0922:TLSC $\mathrm{AH}>2.0 . \mathrm{CO} ; 2$

Luo H, Yanai M (1984) The large-scale circulation and heat sources over the Tibetan Plateau and surrounding areas during the early summer of 1979. II. Heat and moisture budgets. Mon Weather Rev 112:966-989. doi:10.1175/1520-0493(1984)112<0966:TLS $\mathrm{CAH}>2.0 . \mathrm{CO} ; 2$

Masson V, Joussaume S (1997) Energetics of the 6000-yr BP atmospheric circulation in boreal summer, from large-scale to monsoon areas: a study with two versions of the LMD AGCM. J Clim 
10:2888-2903. doi:10.1175/1520-0442(1997)010<2888:EOTYB A $>2.0 . \mathrm{CO} ; 2$

Neelin JD, Held IM (1987) Modeling tropical convergence based on the moist static energy budget. Mon Weather Rev 115:3-12. doi:10.1175/1520-0493(1987)115<0003:MTCBOT>2.0.CO;2

Neff U, Burns SJ, Mangini A, Mudelsee M, Fleitmann D, Matter A (2001) Strong coherence between solar variability and the monsoon in Oman between 9 and $6 \mathrm{kyr}$ ago. Nature (Lond UK) 411:290-293. doi: $10.1038 / 35077048$

Nitta T (1983) Observational study of heat sources over the eastern Tibetan Plateau during the summer monsoon. J Meteorol Soc Jpn 61:590-605

Prell WL (1984) Climate processes and climate sensitivity. In: A response to changing solar radiation. American Geophysical Union, Variation of Monsoonal Upwelling

Prell WL, Kutzbach JE (1992) Sensitivity of the Indian monsoon to forcing parameters and implications for its evolution. Nature 360:647-652. doi:10.1038/360647a0

Scheffer M, Bascompte J, Brock WA, Brovkin V, Carpenter SR (2009) Early-warning signals for critical transitions. Nature 461:53-59

Schellnhuber HJ (2009) Tipping elements in the Earth system. Proc Nat Acad Sci USA 106:20561-20563. doi:10.1073/ pnas.0911106106
Schewe J, Levermann A, Cheng H (2011) A critical humidity threshold for monsoon transitions. Clim Past Discuss 7:1737-1765. doi:10.5194/cpd-7-1737-2011

Spivak M (1965) Calculus on manifolds. Addison-Wesley, Boston

Trenberth KE, Stepaniak DP (2003) Seamless poleward atmospheric energy transports and implications for the Hadley circulation. J Clim 16:3706-3722. doi:10.1175/1520-0442(2003)016<3706:SPA $\mathrm{ETA}>2.0 . \mathrm{CO} ; 2$

Trenberth KE, Stepniak DP, Caron JM (2000) The global monsoon as seen through the divergent atmospheric circulation. J Clim 13:3969-3993. doi:10.1175/1520-0442(2000)013<3969:TGMA $\mathrm{ST}>2.0 . \mathrm{CO} ; 2$

Webster PJ, Magana VO, Palmer TN, Shukla J, Tomas RA, Yanai M, Yasunari T (1998) Monsoons: processes, predictability, and the prospects for prediction. J Geophys Res 103:14451-14510. doi:10.1029/97JC02719

Zickfeld K, Knopf B, Petoukhov V, Schellnhuber HJ (2005) Is the Indian summer monsoon stable against global change? Geophys Res Lett 32(L15):707. doi:10.1029/2005GL022771 